\title{
Building the Global Bioanalysis Consortium - working towards a functional globally acceptable and harmonized guideline on bioanalytical method validation
}

“...it was decided to set-up an all-inclusive Global Bioanalysis Consortium consisting of scientific associations with worldwide recognition and influence in order to create one unified document that, after thorough discussion in global conferences, can be presented to the decision makers in each country or region."

Keywords: bioanalysis $\approx \mathrm{GBC} \approx$ global $\approx$ Global Bioanalysis Consortium $\approx$ guidelines $\approx$ harmonization $\approx$ health authorities $\approx$ pharma

Although harmonization of bioanalytical method validation (BMV) and application has been discussed at scientific forums on several occasions, it was not until the European Bioanalysis Forum (EBF) conference in December 2009 [1], where the European Medicines Agency's (EMA) intended draft bioanalytical method validation guideline [101] was discussed, that the bioanalytical scientists present expressed their strong wish for international harmonization. CT Viswanathan (US FDA/Center for Drug Evaluation and Research [CDER]) and J Welink (Dutch Medicines Evaluation Board [MEB] for EMA), present at the meeting, acknowledged this need for harmonization and encouraged representatives of several international organisations - American Association of Pharmaceutical Scientists (AAPS), Applied Pharmaceutical Analysis (APA), Calibration Validation Group (CVG) and EBF - to work with their respective memberships and collaborate on global BMV harmonization efforts. Specifically, both agency representatives said they would be open to hearing from the international scientific community proposals for consistent harmonized regulatory language.

The first action in this direction was to publish the open letter that they had sent to the US FDA and EMA in February 2010, requesting global harmonization of existing or emerging guidances for BMV and sample analysis and offering to support the processes needed [2]. In addition, representatives of the above named organizations published two editorials in the same journal discussing the history of BMV and expressing their views on a future harmonization process $[3,4]$.

Concurrent with these publications, the CVG had its fourth workshop on regulated bioanalysis in Montreal. This meeting focussed serious attention on the topic of harmonization of bioanalytical guidelines through a number of speakers from industry and regulatory agencies presenting their ideas and discussing possible future processes with the audience [5]. At the CVG workshop, a unanimous consensus was reached among the five regulatory agencies, the panellists and the international audience that global guidance should be science driven, not prescriptive, and should include rationale behind each requirement to prevent 'box checking' by auditors and reviewers of filings. Furthermore, the need to rise above local issues and focus on globally acceptable language will be pivotal to a successful outcome.

\section{"The intention and expectation is that the document must be influential enough that all agencies will want to adopt it and harmonize their auditing and reviewing approaches."}

To transfer words into actions, it was decided to set-up an all-inclusive Global Bioanalysis Consortium (GBC) consisting of scientific associations with worldwide recognition and influence in order to create one unified document that, after thorough discussion in global conferences, can be presented to the decision makers in each country or region. The intention and expectation is that the document must be influential enough that all agencies will want to adopt it and harmonize their auditing and reviewing approaches.

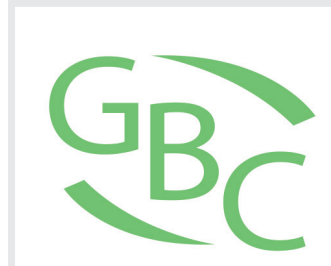

Peter van Amsterdam ${ }^{\dagger 1}$, Mark Arnold², Surendra Bansal $^{3}$, Douglas Fast ${ }^{4}$, Fabio Garofolo ${ }^{5}$, Steve Lowes ${ }^{6}$, Philip Timmerman ${ }^{7}$ \& Eric Woolf ${ }^{8}$

'Abbott Healtcare Products BV, CJ van Houtenlaan 36, I38I CP

Weesp, The Netherlands

${ }^{2}$ Bioanalytical Sciences, Bristol-

Myers Squibb Co., NJ, USA

${ }^{3}$ Hoffmann-La Roche, Inc., NJ, USA

${ }^{4}$ Bioanalytical Sciences, Covance Laboratories, WI, USA

${ }^{5}$ Bioanalytical Services - Algorithme Pharma Inc., Quebec, Canada

${ }^{6}$ Advion BioServices, NY, USA

7 Janssen Research \& Development,

a division of Janssen Pharmaceutica,

Belgium

${ }^{8}$ DMPK-Regulated Bioanalysis, Merck Research Labs, PA, USA

${ }^{\dagger}$ Author for correspondence:

Tel.: +3l 294477691

Fax: +3| 294477 |4|

E-mail: peter.vanamsterdam@

abbott.com 
Following the April 2010 CVG meeting in Montreal, the authors came together in a number of teleconferences to lay the foundations of the GBC and take the first steps in building it. This group of founding members set the following goals and objectives for the GBC:

- Bring together stakeholders from the pharmaceutical industry, contract research organizations and academia to share current understanding of bioanalysis guidelines, identify differences in these guidelines, or differences in the interpretation or application thereof, to routine bioanalysis;

- Come forward with recommendations to health authorities and regulatory bodies worldwide on globally agreed best practices for BMV and application of such methods/technologies to the analysis of drugs of all molecular sizes in support of clinical and nonclinical studies;

- Invite relevant stakeholders, from industry, academia, health authorities and regulatory bodies, to jointly discuss the GBC recommendations at a global conference in order to achieve globally agreed guidelines on bioanalysis;

- Going forward, to serve as a pivot point on the continued harmonized interpretation and/or updates of globally agreed guidelines.

Next, we designed a structure for the Consortium that could be effective: small dedicated teams that give opportunity for scientists from all over the world to participate. In essence, the plan is to set up a steering committee (SC) to guide the process of harmonization, and a number of harmonization teams (HT), to discuss different validation or sample analysis topics. The SC will consist of one or two representatives of every major region and their task will be to:

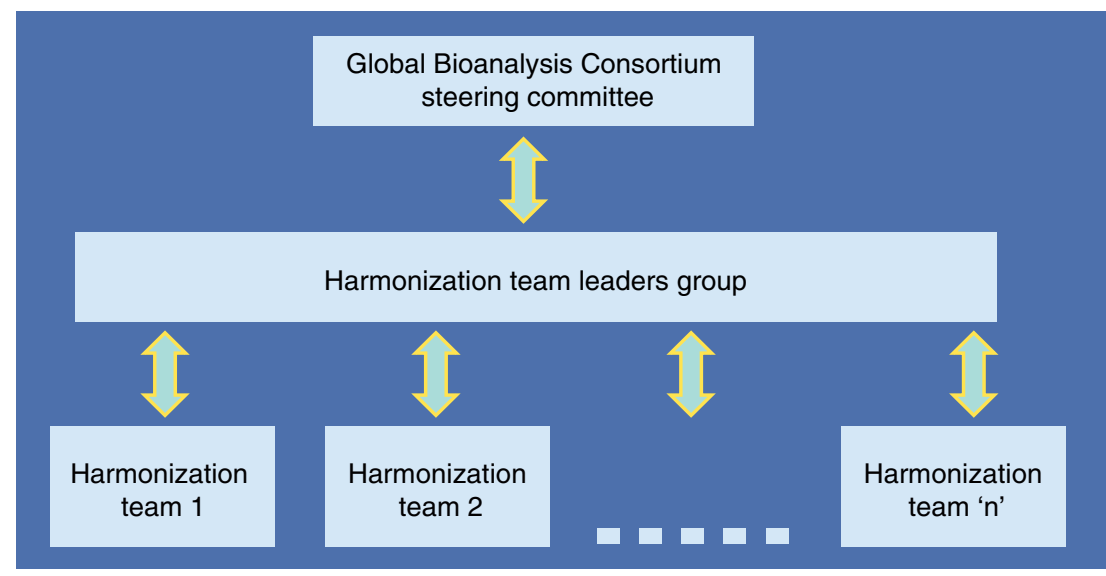

Figure 1. Conceptual organization of the Global Bioanalysis Consortium.
- Coordinate the GBC process of supporting a global BMV harmonizing strategy;

- Assign harmonization team leaders (see following dicussion);

- Connect with and support the harmonization teams working on specific topics;

- Be responsible for communication of the GBC progress to the global community;

- Represent the GBC at international meetings;

- Interact with health authorities/regulatory agencies and organize harmonization international meetings/conferences.

It is important to note that the SC will not own content of harmonization discussion, but can act as a sounding board. Also, the size of this committee will be kept minimal, but all major regions should be represented. Currently four regions are identified: Europe, North America, Latin America and Asia-Pacific.

Connected to the GBC SC will be a group of harmonization team leaders who will be working with their teams on specific topics. They will build and lead the harmonization teams which will work on a number of specific method validation or sample analysis topics. The harmonization team leaders will be responsible for:

- Assigning the HT members from the experts volunteering or experts that have been identified in SC-HT leaders meetings;

- Organizing the HT meetings and documenting the discussions;

- Connecting with SC to report back on progress and/or get input from;

- Connecting with other HT leads in case of topic overlapping discussions.

The real work on the content will be achieved by the harmonization teams. These consist of the above mentioned HT lead and subject matter experts from multiple regions. In those teams, typically a group of five to ten experts, all aspects relevant for a specific bioanalytical topic will need to be thoroughly discussed and prepared for a broader presentation in a global harmonization meeting bringing together industry, academia and interested health authorities. The teams will need to be aware of what historic discussions have occurred over the past 20 years, what was achieved so far and why we came to those decisions. An overview of the GBC team dynamics is given in FIGURE I. 
There are multiple ways to divide the house of bioanalysis in smaller rooms in which only one specific topic will fit while at the same time, it is almost impossible to avoid any overlap from topic to topic. Especially integrating 'macromolecules/ligand-binding assay' with 'small molecules/LC-MS' is going to be a serious challenge. For example, a possible setup in harmonization teams could be as follows:

\section{- General}

- Run acceptance criteria

- Sample stability

- ISR

- Aspects of method transfer

- Bioanalytical reports

- Reference standards, quality controls and reagents

- Quality regime/tiered approach

\section{Small molecules/LC-MS specific}

- Calibration, accuracy and precision

- Sensitivity, specificity and matrix effects

- Metabolite quantification

- Macromolecules/LBA specific

- Calibration, accuracy and precision

- Sensitivity, specificity and matrix effects

- Immunogenicity

The founding members will continue to build the GBC in the coming months and will present their plans at bioanalytical meetings. It is our intention to give full attention to global harmonization and the GBC organisation at the end of the year. More specifically for North America, at the annual AAPS meeting in New Orleans in November [102]; for Europe, at the EBF open meeting in Barcelona in December [103]; and for Asia-Pacific, at the CVG meeting in Shanghai

\section{Bibliography}

1 Abbott R. European Bioanalysis Forum, 2nd Open Symposium: The broadening scope of validation: towards best practices in the world of bioanalysis. Bioanalysis 2(4), 703-708 (2010).

2 Timmerman P, Lowes S, Fast DM, Garofolo F. Request for global harmonization of the guidance for bioanalytical method validation and sample analysis. Bioanalysis 2(4), 683 (2010).

3 Bansal S, Arnold M, Garofolo F. International harmonization of bioanalytical guidance. Bioanalysis 2(4), 685-687 (2010). in January 2011 [104]. In those meetings, representatives from the already assigned GBC SC or one of the founding members will speak and seek input from the broader audience. It is our target to have identified and assigned bioanalytical experts to the SC as HT leads and team members in February 2011. Furthermore, we aim to have a GBC website live in the fourth quarter of 2010.

We expect, and have already recognized, that many bioanalytical scientists are eager to join the initiative and we would like to welcome you all. However, we are intent on building the Consortium as a group truly representing all regions while at the same time we will need to limit the team sizes to achieve results and avoid big groups that will be hampered by size. We fully recognize that this approach may not present all views, but look to the regional organizations to establish and maintain meetings in which broad participation in topical discussions can occur. Until a formal call for participation is issued, if you would like to express your interest in actively participating, feel invited to contact any of the authors of this article at one of the future meetings.

\section{Disclaimer}

The views expressed in this article are those of the Global Bionanalysis Consortium and do not necessarily reflect the respective company's position on the subject.

\section{Financial \& competing interests disclosure}

The authors have no relevant affliations or financial involvement with any organization or entity with a financial interest in or financial conflict with the subject matter or materials discussed in the manuscript. This includes employment, consultancies, honoraria, stock ownership or options, expert testimony, grants or patents received or pending, or royalties. No writing assistance was utilized in the production of this manuscript.
4 van Amsterdam P, Lausecker B, Luedtke S, Timmerman P, Brudny-Kloeppel M. Towards harmonized regulations for bioanalysis: moving forward! Bioanalysis 2(4), 689-691 (2010).

5 Savoie N, Garofolo F, van Amsterdam P et al. 2010 White Paper on recent issues in regulated bioanalysis and global harmonization of the bioanalytical guideline - from The 4th Calibrationand Validation Group Workshop. Bioanalysis DOI:10.4155/BIO.10.164 (2010) (In Press).

\section{- Websites}

101 EMA Draft Guideline on validation of bioanalytical methods. www.ema.europa.eu/pdfs/human/ ewp/19221709en.pdf

102 FIP Pharmaceutical Sciences 2010 World Congress/AAPS Annual Meeting and Exposition. www.pswc2010.org/

103 EBF 3rd Open Conference: From Challenges to Solutions. www.europeanbioanalysisforum.eu/ conferences.php

104 The 1st Workshop in Asia Pacific on Recent Issues in Regulated Bioanalysis. www.canadianlcmsgroup.com/bioanalysis_ asia_workshop.php 